\title{
Die Wunden Jesu - Soteriologische Anmerkungen zu Christus, dem Retter der Verwundeten
}

Martina Bär

Kein Tag vergeht, an dem nicht von Kriegsopfern oder von anderen Gewaltopfern, auch Opfern von >Naturgewalten « die Rede ist. Durch die Flüchtlinge, die aus der südlichen und östlichen Hemisphäre zu uns nach Europa kommen, sind die durch Krieg und Flucht verursachten Verwundungen Gegenstand unserer gesellschaftlichen und politischen Auseinandersetzung geworden. Dabei scheinen die vom Krieg Getriebenen und Verwundeten eine solche Bedrohung darzustellen, dass der Rechtspopulismus fast über Nacht wieder salonfähig geworden ist. Seit der Inauguration Donald Trumps zum Präsidenten der Vereinigten Staaten im Januar dieses Jahres erfahren hiesige rechtspopulistische Parteien zusätzlich starken Rückenwind, denn auch er und seine Hintermänner entpuppen sich als Politiker mit solcher Gesinnung. Man fragt sich, wo die humanitären Werte geblieben sind, wenn hier wie dort Grenzzäune und Mauern errichtet werden und wurden, um den Flüchtlingsstrom abzuwehren. Über solche Problemlösungsstrategien empören sich vor allem diejenigen, die eine Sensibilität für Menschenrechtsverletzungen haben, die die unantastbare Würde des Menschen verteidigen oder für die humanitäre Hilfe eine unbedingte Handlungsmaxime ist. In Europa sind das oft auch jene Menschen, die direkt oder indirekt von den zwei grossen Weltkriegen des vergangenen Jahrhunderts betroffen sind. Sie wissen um den Wert der freiheitlichen Grundrechte und einer Gesellschaft, die diese verteidigt. Die Generationen allerdings, die ohne Kriege aufgewachsen sind - immerhin leben wir Westeuropäer seit mehr als 70 Jahren in Frieden - und die sich auch nicht von anderen, fremden Kriegen betreffen lassen möchten, sind schneller dabei, sich rechtspopulistisches und ausländerfeindliches Gedankengut anzueignen, ohne Mitgefühl für die Flüchtlinge und ohne die Auswirkungen ihrer politischen Einstellung abschätzen zu können. Der Grund hierfür liegt oft in der Angst vor Verlust an Wohlstand oder kultureller Identität. Angst ist aber immer ein schlechter Ratgeber.

In Anbetracht der Notsituationen wäre aber eigentlich jetzt der historische Moment gekommen, in dem die wohlhabenden westeuropäischen Gesellschaften, die durch die Weltkriege des 20. Jahrhunderts leidgeprüft sind und die Verletzungen dieser Kriege gröss- 
tenteils überwunden haben, den Menschen solcher humanitären Katastrophen eine helfende Hand reichen. Zu helfen bedeutet, sich mit hilfsbedürftigen Menschen, die grosse Not und viel Leid erlebt haben, auseinanderzusetzen. Das ist nicht unbedingt einfach. Denn die Menschen, die aus den Kriegsgebieten kommen, sind teilweise schwer traumatisiert. Denjenigen, die hier vor Ort Hilfestellung leisten oder sich auch nur für die Schicksale dieser Menschen interessieren, stockt der Atem, wenn auch nur ein Kriegsflüchtling das Ausmass der erlittenen Katastrophe schildert.

Aus der psychologischen Forschung ist bekannt, dass traumatische Erfahrungen ihre Spuren als posttraumatisches Belastungssyndrom hinterlassen können - vielleicht ein Leben lang, wenn nicht ausreichend Resilienz oder therapeutische Unterstützung vorhanden war, um das Trauma zu heilen. Nicht geheilte Traumata werden an die nächste und sogar übernächste Generation weitergegeben. Dieses Phänomen wurde zunächst bei den Kindern und Enkelkindern der Shoah-Überlebenden beobachtet. Seit ein paar Jahren sind in entsprechender Weise die Kinder der deutschen Kriegsgeneration und sogar die Kriegsenkel zum Gegenstand psychologischer und historischer Forschung geworden. Ein Blick in die Literatur legt in erschreckender Weise offen, wie auch hier nicht-geheilte Traumata von Generation zu Generation weitergereicht werden können. ${ }^{1}$ Fast scheint es so, als ob die Wunde ein Eigenleben führt und bereit ist, viele Jahre und über mehrere Generationen hinweg zu warten, bis sie gesehen wird und geheilt werden kann. Wenn man sich diese Langzeitwirkung von Traumata, also seelische Wunden, vor Augen führt, bekommt man ein Gespür dafür, welchen humanitären Beitrag eine Gesellschaft für das Wohl der Menschheitsfamilie leistet, wenn sie bereit ist, sich in materieller und psychologischer Hinsicht um (Kriegs-)Opfer zu kümmern. Es wird nicht nur jeweils ein Mensch gerettet, vielmehr trägt dessen Rettung ebenso zum Wohl der ihm nachkommenden Generationen bei und ermöglicht damit eine intakte Gesellschaft aus sheilen Mitgliedern. Darüber hinaus kann die Trauma-Heilung auch mit einer Aussöhnung zwischen Opfer und Täter einhergehen. Hatte Gott diese Dimension im Blick, als er sich selbst in Jesus v. Nazareth zu unserem Heil verwundbar machte?

1 Vgl. dazu Anne-Ev Ustorf, Wir Kinder der Kriegskinder. Die Generation im Schatten des Zweiten Weltkriegs, Freiburg i.Br. ${ }^{4} 2013$; Udo Baer/Gabriela FrickBaer, Wie Traumata in die nächste Generation wirken. Untersuchungen, Erfahrungen, Therapeutische Hilfe, Neukirchen-Vluyn 2010; Udo Baer/Gabriela Frick-Baer, Kriegserbe in der Seele. Was Kindern und Enkeln der Kriegsgeneration wirklich hilft, Weinheim 2015; Sabine Bode, Die vergessene Generation. Die Kriegskinder brechen ihr Schweigen, Stuttgart ${ }^{30} 2016$. 
Jesus v. Nazareth lebte als Mensch unter menschlichen Bedingungen, was für unser Thema bedeutet: Er war verwundbar wie wir es sind. Er beliess es nicht bei der Potentialität, sondern liess sich freiwillig verwunden und machte sich zum Opfer. Die Wunden Jesu reduzieren sich nicht auf die zugefügten Wunden durch die Kreuzigung. Er war auch Folteropfer, fühlte sich mit hoher Wahrscheinlichkeit am Kreuz gottverlassen, erlebte die Agonie im Garten Gethsemane. $\mathrm{Zu}$ ertragen hatte er, dass er unschuldig verurteilt und hingerichtet worden ist und dabei von seinen Freunden allein gelassen worden war. Heute würden wir aufgrund der zugefügten Qualen mutmassen: Auch Jesus müsste Traumatisierungen erlitten haben. Seine Geschichte endet jedoch nicht mit dem psychischen und physischen Schmerz und Tod, sondern er wird von Gott geheilt und gerettet, indem er zu einem neuen Leben auferweckt wird. Als Auferweckter, der noch die Stigmatisierungen seiner erlittenen Gewalt trägt, wird er fortan von seinen Jüngerinnen und Jüngern als der Messias - der Retter - verkündet. Diese eigenartige Mischung aus bleibender Stigmatisierung, Auferstehungsleib und Würdetitel regen zum Nachdenken über Jesus Christus als den Retter der Verwundeten an. Was heisst das konkret für die Heilung menschlicher Wunden? Haben die Wunden Jesu erlösendes Potential? Wenn ja, inwiefern hat das Sich-Verwundbar-Machen Gottes in den Verwundungen Jesu einen soteriologischen Sinn? Zur Beantwortung dieser Frage ist ein Blick auf die Satisfaktionslehre des Anselm v. Canterbury (1033-1109) unumgänglich, da sie das Erlösungshandeln Gottes auf das Kreuz verengte und die Soteriologie stark beeinflusste. In einem zweiten Schritt werden die biblischen Antworten auf die Frage nach dem soteriologischen Sinn des Leidens und Sterbens Jesu skizziert. Schliesslich wird in Anlehnung an Thomas Pröppers Soteriologie die These entfaltet, dass Gottes Liebe der Grund für das Sich-Verwundbar-Machen Gottes in der Welt ist. Gottes Liebe ist die pneumatologisch präsente Kraft, die es auch heute vermag, Wunden zu heilen und einen Menschen zu transformieren.

\section{Satisfaktionslehre und Kritik}

Die Frage nach dem soteriologischen Sinn des Sich-VerwundbarMachen Gottes ist heikel, weil Anselm v. Canterbury in Cur deus $h^{\prime} o^{2}$ (ca. 1094) den Opfertod und das Kreuz mit Heil identifi-

2 Anselm v. Canterbury, Cur deus homo - Warum Gott Mensch geworden, lateinisch-deutsch, hg. und übersetzt von Franciscus Salesius Schmitt (OSB), Darmstadt 
zierte und das Erlösungshandeln Gottes in Jesus v. Nazareth darauf reduzierte. In den letzten Jahrzehnten ist diese Soteriologie auf viel theologische Kritik gestossen, weil die Argumentation theologisch problematisch und historisch nicht zutreffend ist. Ausserdem entfaltete sie eine Wirkungsgeschichte mit negativen Folgen für die Gläubigen. ${ }^{3}$ Kritische Vordenker dieser systematisch-theologischen Kritik waren Freiheitsphilosophen der Neuzeit wie Hegel und im Anschluss daran Religionskritiker wie Marx, Feuerbach oder Nietzsche. Die historische und philosophische Kritik brachte ans Licht, dass sich die anselmische Deutung des Opfertodes Jesu nicht vereinbaren lässt mit der jesuanischen Rede vom barmherzigen Gott oder mit der Eigenschaft der Güte Gottes im Sinne von Gott als höchstem Gut und Ursprung aller innerweltlichen Gutheit. Anselm entfaltete unter Anwendung des in der Theologie bereits adaptierten römisch-/germanisch-feudalen Rechtsdenkens, das dem Prinzip der ausgleichenden Gerechtigkeit zur Tilgung einer Schuld folgt, die Idee, dass Gott selbst dieses Kreuzesopfer zur Wiederherstellung seiner Ehre benötigte. Schuld daran ist der Mensch, der durch die Sünde Gott die geschuldete Anerkennung entzogen habe, ihn seiner Ehre beraubte und so die gerechte Weltordnung gestört habe. Der Mensch ist Gott die Ehrerbietung schuldig, denn immerhin ist Gott sein Herr und Erhalter seiner Lebensgrundlage. Die Gerechtigkeit Gottes (im Sinne des römisch-germanischen Rechtsdenkens) verlangt es, dass der Mensch eine freiwillige und angemessene Wiedergutmachung leistet, wenn er nicht der verdienten Strafe verfallen möchte. Denn auf jede Sünde muss nach Anselm Strafe oder aber Genugtuung folgen. ${ }^{4} \mathrm{Um}$ die Menschen nicht bestrafen zu müssen - und darin zeigt sich die Güte Gottes gegenüber den Menschen -, opfert Gott seinen Sohn, einen Gottmenschen ohne Sünde, stellvertretend für die Menschen. Somit ist Genugtuung geleistet, die Schuld ist abgegolten und die rechte Ordnung ist wiederhergestellt. Die erforderliche Genugtuung kann in der Logik Anselms nur Jesus bringen, weil die Menschen zu sehr in der Erbschuld Adams verstrickt waren, als dass sie angemessene Genugtuung hätten leisten können.

\footnotetext{
${ }^{4} 1986$.

3 Vgl. dazu etwa: Thomas Pröpper, Erlösungsglaube und Freiheitsgeschichte. Eine Skizze zur Soteriologie, München ${ }^{3} 1991$; Hans Kessler, Die theologische Bedeutung des Todes Jesu. Eine traditionsgeschichtliche Untersuchung, Düsseldorf ${ }^{2} 1971$; eine Zusammenführung seiner langjährigen Forschung zu diesem Thema gibt Jürgen Werbick, Gott - menschlich. Elementare Christologie, Freiburg/Basel/Wien 2016.

4 Vgl. Cur deus homo, 51; I,15.
} 
Anselm bindet die Barmherzigkeit Gottes an die Gerechtigkeit. In seiner Denkweise gibt es keine bedingungslose Barmherzigkeit. Fatal an Anselms Satisfaktionslehre ist zudem, dass sie eine soteriologische Verengung auf das Kreuz erzeugte und nur ihm schuldtilgende Bedeutung beimass. Die bedingungslose Liebe und Vergebung Gottes, wie sie Jesus verkündigt hat - man denke nur an das Gleichnis des verlorenen Sohns oder die Sündenvergebungspraxis Jesu, die ohne Tempelkult und Opfer auskam - verloren ihren Sinn. Der Bezug zum Evangelium Jesu und seiner Gottesverkündigung war nicht mehr gegeben. Die Genugtuungslehre gewann trotz ihres offenkundigen Widerspruchs zur Botschaft Jesu schnell an Einfluss, auch wenn sie im Mittelalter teilweise kritisiert (Abälard) oder wie bei Thomas v. Aquin durch Hinzufügung weiterer Gründe für die Inkarnation Gottes als Mensch relativiert worden ist. Es ist gerade auf die Vermittlung von Thomas zurückzuführen, dass diese Konzeption der Erlösung die Folgezeit derart beeinflusste, dass sie in der katholischen Theologie bis heute präsent ist. Aber auch in der altprotestantischen Dogmatik sowie der evangelisch-konfessionellen Theologie des neunzehnten Jahrhunderts hat sie eine bedeutende Rolle gespielt. ${ }^{5}$ Die Implikationen der Satisfaktionslehre haben einen tiefen Eindruck in dem Schuldbewusstsein der Christinnen und Christen hinterlassen; sie fühlen sich beispielsweise schuldig für den Tod Jesu. Die Satisfaktionslehre belastet zudem das Gottesverhältnis, weil sie dazu tendiert, das Gottesbild zu dämonisieren: »Man wendet sich mit Schrecken von einer Gerechtigkeit ab, deren finsterer Zorn die Botschaft von Liebe unglaubwürdig macht. « ${ }^{6}$ Diese Analyse basiert auf Feuerbachs treffender Kritik an der anselmischen Deutung von der Notwendigkeit der Inkarnation und des Kreuzestods. Er fragte, ob nicht hinter der Liebe Gottes eine allmächtige Willkür lauert, vor der man sich ängstigen muss, wenn Gott Gefallen an einem Genugtuungsopfer hat. Feuerbach opferte deswegen lieber Gott, den er als "das böse Wesen des religiösen Fanatismus " ${ }^{7}$ bezeichnete, um die menschliche Liebe zu retten.

Ein weiteres Problem, das durch die Isolierung des Kreuzes als Heilsereignis entstand, war, dass das singuläre Leiden Jesu verklärt worden ist. Daraus entstand eine generelle Verklärung des Leidens: allem Leiden wurde eine Sinnhaftigkeit zugesprochen. ${ }^{8}$ Die Schat-

5 Vgl. Wolfhart Pannenberg, Grundzüge der Christologie, Gütersloh ${ }^{3} 1969,37,287 \mathrm{f}$.

6 Joseph Ratzinger, Einführung in das Christentum, München 1968, 231.

7 Ludwig Feuerbach, Das Wesen des Christentums [1841], Frankfurt a.M. 1975, 61.

8 Vgl. Dietrich Wiederkehr, Glaube an Erlösung. Konzepte der Soteriologie vom Neuen Testament bis heute, Freiburg i.Br. 1976, 56. 
tenseite davon ist, dass man dem Leiden, das hätte beendet werden können, eine religiöse Bedeutung beigemessen hat. Es wurde sinnlos fortgeführt oder sogar potenziert. Sinnlos deswegen, weil Gott doch vom Leiden befreien wollte. Das Leiden wurde aber aufgrund der Kreuzesverherrlichung als gottgewollt hingestellt und legitimierte konkrete Opfer religiös mit dem Argument, Gott würde dies gefallen. Auch konnten so lange die Täter, die Mitmenschen verletzten und zwischenmenschliches Leid und Traumata verursachten, mit einer religiösen Legitimation gedeckt oder entschuldigt werden. Die individuelle Verantwortung eines Täters für seine Tat wurde so verwischt und notwendige zwischenmenschliche Versöhnungsprozesse verhindert.

Die Feministische Theologie und die Befreiungstheologie, die aus der Perspektive der Unterdrückten, Armen und Opfer Theologie betreiben, haben aus diesen Gründen die durch die Satisfaktionslehre hervorgerufene Staurozentrik in der Soteriologie kritisiert, weil eine Fixierung auf das Kreuz und Opfer Jesu wenig erlösendes Potential für diejenigen hat, die auf derVerliererseite des Lebens stehen. Vielmehr wirkt die Kreuzesverklärung wie eine Versöhnung Gottes mit dem Elend und somit als geeignetes Mittel der Vertröstung. ${ }^{9}$ Für sie kann die Verherrlichung eines Lebens in Schmerzen, Ohnmacht, Freudlosigkeit und Unterdrückung schlechterdings nicht das Evangelium Jesu Christi sein, das ein Leben in Fülle verheisst. Sie haben auf den Ereigniszusammenhang von Tod und Auferweckung verwiesen. Das Kreuz Jesu bedeutet für sie, dass die ganze Schöpfung solidarisch "Nein zu den Mächten sagt, die Menschen töten « ${ }^{10}$. Ferner verweist Jesus am Kreuz auf die Solidarität Gottes mit den Opfern, besonders mit jenen, die sich für die Gerechtigkeit (das Reich Gottes) in der Welt einsetzen und dafür sterben mussten. Das Kreuz lädt auch zum Kampf gegen die Herrschaft der todbringenden Mächte ein, denn es ist eine prophetische Anklage an die Mächtigen, die den Tod Unschuldiger verursachen. Die Befreiungstheologien sehen in der erlittenen Ohnmacht Jesu das Gegenteil von Verharrung in Ohnmacht, weil sie Kreuz und Auferweckung Jesu zusammendenken. Mit der Auferweckung schenkt Gott nicht nur neues Leben, sondern führt den weltlichen und todbringenden

\footnotetext{
9 Vgl. Thomas Pröpper, Erlösungsglaube und Freiheitsgeschichte, 94; ausführlicher: Jürgen Moltmann, Der gekreuzigte Gott. Das Kreuz Christi als Grund und Kritik christlicher Theologie, München 1972, 47-52; Dorothee Sölle, Leiden, Stuttgart/ Berlin 1973, 17-44.

${ }^{10}$ Ivone Gebara, Die dunkle Seite Gottes. Wie Frauen das Böse erfahren, Freiburg i.Br. 2000, 151.
} 
Mächten auch die Gerechtigkeit des Reiches Gottes vor Augen und richtet sie wieder auf. Die Auferstehung ist der Triumph der Gerechtigkeit, der "vorweggenommene Anbruch der endgültigen Befreiung" (Leonardo Boff). Die Auferstehung befreit Menschen dazu, nicht mehr "Sklaven" der Geschichte zu sein, sondern die Geschichte selbst in die Hand zu nehmen und bereits in der Geschichte als Auferstandene zu leben und die unzerstörbare Hoffnung, Freiheit und Freude Gottes widerzuspiegeln.

Neben den Befreiungstheologien haben auch viele andere systematische Theologen die soteriologische Verengung der Kreuzestheologie kritisiert und das Heilsereignis wieder, wie im Frühen Christentum und in der Alten Kirche üblich, als einen Ereigniszusammenhang von Inkarnation, Leben und Auferweckung Jesu gesehen. Dennoch ist der Glaube, dass die Wunden Jesu eine Heilsbedeutung haben, schon früh im Christentum verankert. Im Neuen Testament gibt es eine wirkmächtige Tradition, die den Wunden Jesu und seinem Tod einen besonderen Sinn verleiht, indem ihnen Heilsbedeutung für uns Menschen zugesprochen wird. Was sagen die neutestamentarischen Quellen über die Verwundungen und das Opfer Jesu zum Heil der Menschen?

\section{Der Sinn des Leidens und Sterbens Jesu im Neuen Testament}

Die ersten Christinnen und Christen suchten nach einer Erklärung für die Passion Jesu Christi. Warum musste Jesus, der Gerechte, leiden, und warum am Kreuz sterben? Heute würden wir aus theologischphilosophischer Perspektive sagen, dass damals eine Antwort auf die Theodizeefrage gesucht wurde: Warum hat der gerechte und moralisch gute Gott diese Ungerechtigkeit zugelassen und Jesus sinnlos sterben lassen? Aus welchen Gründen hat Jesus sich nicht gewehrt und hat die Gewalt und den Tod freiwillig über sich ergehen lassen? Warum hat er sich selbst zum Opfer gemacht? Da der in Israel präsente Tun-Ergehen-Zusammenhang im Falle Jesu nicht greift, da er als Gerechter gestorben ist, wurde die Frage schnell mit Gott in Verbindung gebracht und nach einem tieferen, religiösen Sinn für dieses Leiden und Sterben gesucht. Dieser tiefere Sinn wurde in den Sühneopfervorstellungen und der Typologie des leidenden Gerechten gefunden. Das Neue Testament bekennt deshalb, dass Jesus nicht nur ein Opfer eines ungerechtfertigten Urteils von römischen und jüdischen Machthabern gewesen ist (victim), sondern auch ein Opfer 
für die Rettung und Heilung der Menschen (sacrifice). ${ }^{11}$ Die ersten Christinnen und Christen glaubten, dass Gott aus dem politischen victim ein sacrifice gemacht hat und so die umfassende Versöhnung zwischen Gott und Mensch bewirkt hat. Dies gilt als Heilstat, denn sie hat zur Folge, dass die Lebensgemeinschaft zwischen Gott und den Menschen endzeitlich gültig erneuert ist. Das sacrifice Jesu steht im Zusammenhang mit der Reiches-Gottes-Sendung und mit der damit einhergehenden Dringlichkeit zur Umkehr. Es vollendet diese Reich-Gottes-Botschaft in dem Sinne, dass Jesu bereit war, dafür zu sterben. Jesus stimmte die Menschen auf die Liebe Gottes ein und blieb sich, seiner Botschaft und Gott bis zum Tod treu. Der Grund für die Notwendigkeit seines Todes kann folglich

»allein [in] Gottes für den Menschen entschiedene Liebe selbst sein: Weil Gott, frei und sich selber entsprechend, den Menschen von Ewigkeit her erwählt und zur Gemeinschaft bestimmt hat, musste er in der Mitteilung seiner Liebe bis zum Äussersten gehen. \Notwendig` war die Dahingabe seines Sohnes dann im Sinne der Konsequenz seiner Liebe, die in diesem Geschehen ihre Treue zu sich selbst und zum Menschen erwies. ${ }^{12}$

Die Reich-Gottes-Botschaft, die beinhaltet, dass sich Gott den Menschen neu zuwendet, gelangte so an ihr Ziel. Darüber hinaus brachte das Frühe Christentum, das im Umfeld der Synagoge entstand, den Tod Jesu mit jüdischen Sühneopfervorstellungen in Verbindung. Im Folgenden werden diese drei Antwortmodelle kurz skizziert und ihr Verständnis von Heil dargelegt.

\subsection{Synoptische Evangelien}

In den Evangelien ist noch nicht davon die Rede, dass das Kreuz selbst das Heil bewirkt in dem Sinne, dass das Kreuz das Heil des neuen Äons bringt. Auch ist es nicht das Kreuz oder der Tod Jesu, die in die Gottesherrschaft führen oder die Partizipation an ihr ermöglichen. Die synoptischen Evangelien rekurrieren auf die soteriologische Relevanz der Gottesknechtstheologie des Deuterojesaja. "Durch sein Martyrium schafft der Gottesknecht, der Gerechte par excellence, dem Volk - den Vielen - Sühne; ihm sind die Vergehen des Volkes aufgeladen, damit er sie wegtrage und so unschädlich mache. ${ }^{13}$ In Jesaja 53,5 heisst es entsprechend:»Züchtigung für unser Heil lag auf

${ }^{11}$ Vgl. dazu ausführlich:Werbick, Gott-menschlich, 157-212.

12 Ebd., 97.

13 Ebd., 173. 
ihm, durch seine Wunde ward uns Heilung zuteil«. Ein paar Verse weiter heisst es, dass Gott Gefallen an diesem Gottesknecht hat, »der sein Leben als Sühneopfer hingab« (Jes 53, 10). Übertragen auf Jesus bedeutet dies, dass Jesus als der Gerechte schlechthin den Tod eines Sünders stirbt. Jesus hat durch Leiden und Tod die Strafen, welche die Anderen fairerweise verdient hätten, abgewendet. Er sühnte stellvertretend und machte sich darin zum sacrifice. Sühne meint in Israel die Wiedergutmachung eines Vergehens. Denn in Israel wurde meist in der Logik des Tun-Ergehen-Zusammenhangs gedacht: "Wenn einem Menschen, der sich vergangen, der mit einem anderen gebrochen oder etwas Verkehrtes begangen hatte, danach auch selbst Unheil widerfuhr, dann war das für Israel keine Strafe, sondern nur die selbstverständliche, zwangsläufige Folge des eigenen vorangegangenen Fehlverhaltens. ${ }^{14}$ Gott als gerechter Richter "achtete snur darauf, dass die Übeltäter sich nicht einfach den Folgen ihrer Taten entziehen konnten ${ }^{15}$. Dementsprechend heisst es in der Selbstvorstellung Gottes: »Jahwe ist ein barmherziger und gnädiger Gott, langmütig und gross an Güte und Treue, indem er Güte den Tausenden bewahrt [und] indem erVergehen,Verbrechen undVerfehlungen trägt. Aber gewiss entlässt er nicht aus der Haftung, indem er sich um das Vergehen (der) Väter kümmert, (das) auf den Söhnen und Enkeln und auf der dritten und vierten Generation (lastet). ${ }^{16}$ (Ex 34,6 f.) Das heisst, die zugefügten zwischenmenschlichen Verletzungen hat Gott nicht vergessen.Vielmehr erinnert er sich so lange daran (bis in die vierte Generation), bis zwischenmenschlich Sühne geleistet ist, also bis Wiedergutmachung erfolgt ist. Im zwischenmenschlichen Bereich bot man demjenigen, den man verletzt hatte, eine Gabe an, die das Fehlverhalten wieder gut machen sollte. Der Beschädigte verzichtete im Gegenzug auf sein Recht, sich zu revanchieren. ${ }^{17}$

In der Gott-Mensch-Beziehung gab es die durch die Priesterschaft vermittelte Sühneopferpraxis, die einen sühnenden Umgang mit Gott einübte. ${ }^{18}$ In Israel war man sich aber darüber bewusst, dass jede Tat die Gottesbeziehung störte, unabhängig davon, ob eine Tat bewusst, absichtlich oder unbewusst geschah. Das in Levitikus

\footnotetext{
${ }^{14}$ Meinrad Limbeck, Abschied vom Opfertod. Das Christentum neu denken, Ostfildern ${ }^{5} 2013,96$.

${ }^{15}$ Ebd.

16 Übersetzung Meinrad Limbeck, ebd., 97. Limbeck weist darauf hin, dass es im Hebräischen das Wort "Strafe« oder "strafen" gar nicht gibt. Eigentlich steht dort, wo allgemein mit "strafen « übersetzt wird, Haftung für die eigenen Vergehen übernehmen oder selbst die Folgen eines verkehrten Tuns tragen (vgl. ebd., 93-95).

${ }^{17} \mathrm{Vgl}$. ebd., 97.

18 Vgl. ebd., 99.
} 
4,27-30 beschriebene Sühneritual sollte helfen, diese Störung und Belastung der Gottesbeziehung wieder in Ordnung zu bringen. Gegen Gott gerichtete Vergehen würde Gott, so die Theologie der jüdischen Priester, unter der Voraussetzung vergeben, dass "der Schuldige bereit ist, sich einem bestimmten kultischen Akt zu unterziehen, in welchem er seine erneute Zuwendung zu Gott und seine weitere Bindung an Gott dokumentierte « ${ }^{19}$. Am Grossen Versöhnungstag (Jom Kippur) vollzog der Hohepriester stellvertretend für das in Sünde gefallene Volk Israel das Blutopfer. Mit der Applikation des Opferblutes auf den Deckel der Bundeslade, das Hilasterion (hebr.: kapporeth), wurde die Bundeslade, das im Judentum das Allerheiligste ist, neu für die heilbringende Gegenwart Gottes geweiht. Das Blut symbolisiert die Lebenskraft im Fleisch und die Vergiessung des Opferblutes reinigt den Menschen von den Sünden und erneuert seine Lebenskraft. Sühne setzt in diesem Sinne einen Prozess der Erneuerung der Bundesgemeinschaft zwischen Gott und Mensch in Gang. Sie beruht auf der zuvorkommenden Gnade Gottes. Der Opferaltar ist im Alten Testament ein "Ort des Kommens Gottes«; das Opfer ist "Zeichen der Gastfreundschaft gegenüber Gott«. ${ }^{20}$ Gott kommt anlässlich einer Opfergabe, um das Volk zu segnen (Ex 20,24b). Im Unterschied zur Satisfaktionslehre des Anselm von Canterbury braucht in der jüdischen Sühnelogik Gott kein Opfer, um verzeihen und vergeben zu können.Vielmehr unterstützt Gott den Menschen in seinem Bedürfnis nach Wiedergutmachung und Neuanfang, indem er ein Tier zurVerfügung stellt. So kann der Mensch seine neue Lebenshingabe an Gott wirkungsvoll dokumentieren. Der Tod Jesu wird in den Evangelien vor diesem Hintergrund nicht nur als politisch motivierte Hinrichtung betrachtet, sondern auch als sacrifice angesehen, wonach gemäss jüdischer Gottesknechtstheologie das (Opfer-)Blut eines Gerechten stellvertretend für andere Menschen Sühne bewirken kann. Der soteriologische Heilsaspekt des Opfers Jesu liegt demnach auf der Versöhnung zwischen Mensch und Gott, wodurch das Leben der Menschen erneuert worden ist. Das ist etwas, was für unser aufgeklärtes Verständnis schwer nachvollziehbar ist, zumal wir nicht mehr im Horizont jenes Sündenbewusstseins leben.

Die Apostelgeschichte als Teil der lukanischen Theologie macht deutlich, dass der Kreuzestod nicht als Scheitern der Sendung Jesu aufgefasst werden kann. Lukas argumentiert in den Petrusreden (Apg 2,22-24 und 10,34-43) mit dem sogenannten Kontrastmodell. Der

\footnotetext{
${ }^{19}$ Ebd., $99 f$.

${ }^{20}$ Bernd Janowski, Homo ritualis. Opfer und Kult im alten Israel, in: Bibel und Kirche 64 (2009), 134-140, hier $135 \mathrm{f}$.
} 
Kontrast zwischen dem Gerechten und der Ungerechtigkeit seines Todes verdeutlicht, dass das Kreuz eher wals die Vollendung des Lebens und Weges Jesu ${ }^{21}$ verstanden werden muss, denn als Scheitern seiner Verkündigung. Denn mit der Auferweckung Jesu setzte der Vater die Gerechtigkeit des Reiches Gottes, die Jesus verkündigt hat, neu in Kraft, die mit der Hinrichtung Jesu am Kreuz ins Unrecht gesetzt worden war. Die Auferweckung Jesu impliziert die Rettung aller. Die Treue Jesu zur Gerechtigkeit Gottes hatte sich gelohnt und diese Gerechtigkeit siegte.

\subsection{Paulusbriefe und Hebräerbrief}

Paulus war einer der ersten, der im Kreuzestod ein Heilsereignis mit apokalyptischer Wirksamkeit sah und eine spezifische Kreuzestheologie auf dem Hintergrund der apokalyptischen Zwei-Äonen-Lehre entfaltete. Der Alte Ä̀n war beherrscht von weltlichen Mächten, der Sünde und dem Tod; der neue Äon steht unter den Vorzeichen des Sieges über diese Mächte und ist das Zeitalter der Gottesherrschaft. Für Paulus ist »[d]as Kreuz [...] das Ende des alten und der Aufgang des neuen Äons, da mit ihm die liebende Selbsthingabe Gottes in Jesus offenbar wird und aus dem Tod Jesu das Leben im neuen Äon schafft. Der Geist, in dem der Gekreuzigte auferweckt wurde, ergreift die Glaubenden, damit auch sie im Glauben und durch die Taufe zum Leben im neuen Äon - in Christus - auferweckt werden (Röm 6,1-14; vgl. auch Mk 16,16). $\mathbb{1 2}^{22}$ Das Kreuz sieht also zunächst wie der Sieg der Mächte des alten Äons aus, doch in Wirklichkeit ist das Kreuz der Anbruch des neuen Äons. Christus ist der Retter von den Mächten des alten Äons, insofern er den Menschen die Angst vor dem Tod genommen ${ }^{23}$ und gezeigt hat, wie das neue Leben im Geist Gottes geprägt ist, nämlich nicht von der Liebe Gottes geschieden zu sein (vgl. Röm 8,37-39). Darin liegt die durch das Kreuz ermöglichte neue Seinsqualität. Darauf gehe ich später noch einmal ein.

DerTod am Kreuz ist für Paulus ein Opfertod, ein sacrifice gewesen, das Versöhnung mit Gott bewirkt hat. Dies betont auch der Hebräerbrief. Durch dieses sacrifice sind alle rituellen Opfer der Menschen obsolet geworden, da sich Gott im sacrifice Jesu selbst zum Opfer ge-

\footnotetext{
${ }^{21}$ Grundlagentext des Rates der Evangelischen Kirche in Deutschland, Für uns gestorben. Die Bedeutung von Leiden und Sterben Jesu Christi, Gütersloh 2015, 30.

${ }^{22}$ Werbick, Gott-menschlich, 167.

23 "Verschlungen ist der Tod vom Sieg. Tod, wo ist dein Sieg? Tod, wo ist dein Stachel?" (1 Kor 15,54-57; nach Jes 25,8 und Hos 13,14).
} 
macht und den Menschen als Gabe geschenkt hat. Das bedeutet, dass alle menschlichen Versuche, Gott durch eine (Opfer-)Gabe für sich zu gewinnen, überholt sind. Die vielfältige Opferpraxis Israels wird bei Paulus und im Hebräerbrief als "Vorläufer" dessen angesehen, was in Jesus Christus endzeitlich wirksam geschieht. Der bisherige Sühneopferkult mit Tieren ist durch das sacrifice Jesu abgelöst. Paulus (Röm 3) und der Hebräerbrief haben das blutige Kreuzesleiden in Entsprechung zum bisherigen Opferkult als Opferliturgie beschrieben. Das Kreuz ist nach Paulus nun das neue wahre Hilasterion und damit der Ort der gnadenhaften Gegenwart Gottes. Es ist offen in die Welt gestellt, nicht mehr verborgen im Allerheiligsten und nur einmal im Jahr für den Hohepriester zugänglich. »Alle können nun zu Gott Zugang finden [...]. « ${ }^{24}$ Auf den ersten Blick sieht das Kreuz wie ein Ort der äussersten Gottferne aus, in Wahrheit ist es die heilvolle Selbst-Vergegenwärtigung Gottes am Kreuz - und damit Hoffnungszeichen für alle Leidenden. In diesem Sinne ist der Kreuzestod das Versöhnungsangebot Gottes. Dieses Versöhnungsangebot ist heilsam, weil der Mensch von der Gottentfremdung des Alten Äons erlöst ist und die Gemeinschaft auf eine neue Basis gestellt worden ist. ${ }^{25}$

\section{3 »Durch seine Wunden seid ihr geheilt» (1 Petr 2,24) Petrusbrief}

Im Petrusbrief, der als Adressaten eine von Paulus gegründete Gemeinde hatte, taucht die Wendung »Durch seine Wunden seid ihr geheilt" (1 Petr 2, 24) ${ }^{26}$ auf. Wortwörtlich sind hier nicht die Kreuzeswunden gemeint, sondern die Striemen (" $\left.\mu \omega^{\prime} \lambda \omega \pi \iota \ll\right)$, die auf die Folter Jesu anspielen. Diese Striemen-Wunden sind als eine Metapher für die Passion Jesu zu verstehen. Der Verfasser des Pe-

\footnotetext{
${ }^{24}$ Werbick, Gott-menschlich, 211.

${ }^{25}$ Paulus erklärt im Galaterbrief, dass Jesus im Kreuzestod stellvertretend den drohenden Fluch Gottes auf sich genommen hat und die Menschen davor rettete. Das Frühe Christentum judenchristlicher Provenienz befürchtete den Fluch Gottes im Endgericht, denn der Fluch wäre die gerechte Strafe für die menschlichen Vergehen gewesen. Der Kreuzestod, der im Judentum als Tod fürVerfluchte galt (Dtn 21,23), wurde demnach auch im Hinblick auf das Endgericht als heilsrelevant gedeutet. "Christus hat uns vom Fluch des Gesetzes freigekauft, indem er für uns zum Fluch geworden ist; denn es steht in der Schrift: ,Verflucht ist jeder, der am Pfahl hängt‘." (Gal 3,13) Das rettende Heilsprinzip lautet, dass Gleiches mit Gleichem geheilt wird, jedoch in antithetischer Entsprechung. Jesus also, der keine Sünde hatte, hat sich im Kreuzestod zum `Sünder bzw. zum Verfluchten gemacht, um den drohenden Fluch abzuwenden. ${ }^{26}$ Vgl. dazu ähnlich: Jes 53,5.
} 
trusbriefes wollte den Adressaten, die, wie die neutestamentliche Forschung annimmt, unter der Christenverfolgung des Domitian zu leiden hatten, Mut machen und ihr ethisches Verhalten trotz Unterdrückung und Anfeindungen stärken. Sie sollen sich an Christus, der trotz Lauterkeit leiden musste, ein Beispiel nehmen und wie er der Gerechtigkeit Gottes treu bleiben. Es sei besser diesseits für gute Taten zu leiden, als jenseits für böse (1 Petr 3,17). Sie werden wie Jesus in die Herrlichkeit Gottes eingehen, nachdem sie wie er für die Gerechtigkeit des Reiches Gottes Leiden erdulden mussten. ${ }^{27}$ Der Petrusbrief argumentiert schon auf dem Hintergrund der Parusieverzögerung. Er gibt den Wunden Jesu denselben soteriologischen Sinn wie Paulus, wenn er von der Ermöglichung eines neuen Lebens mit Gott spricht, nur mit dem Unterschied, dass die Christenverfolgung als ernstzunehmende Leidensprüfung im Diesseits betrachtet wurde. Die Aussicht auf die Partizipation an der Herrlichkeit Christi im Jenseits konnte helfen, diese Situation zu ertragen. So lautet die uns heute immer noch geläufige Argumentation: Leiden die Christen für die Gerechtigkeit des Reiches Gottes, dann dürfen sie frohen Mutes sein, denn es ist der Wille Gottes. Wie bei Jesus ist auch dieses Leiden nicht umsonst, denn es führt in die Herrlichkeit Gottes. Die Verfolgten und Unterdrückten können in ihren Passionssituationen deswegen sogar Gott für das Leiden verherrlichen (1 Petr 4,16). Hier sehen wir den Beginn einer mit Gottes Willen legitimierte Verherrlichung von Leiden, allerdings zum Zweck der Mitarbeit an der Gerechtigkeit Gottes in der Welt - nicht zum leidensmystischen Selbstzweck.

\section{Gottes Liebe heilt die Wunden}

Die neutestamentlichen Zeugnisse zeigen, dass die ersten Christinnen und Christen durch Passion, Kreuzestod und Auferweckung "den Mut [fanden,Vf.], an einen verwundbaren Gott zu glauben ${ }^{28}$. Von daher hat Dorothee Sölle treffend formuliert: "Christus ist die Wunde Gottes in der Welt «29. Aber heilen nun die Wunden Jesu die menschlichen Verwundungen? Haben die Wunden Jesu konkrete Heilsrelevanz für die Opfer von Gewalttaten? Ich denke, nein. Nicht

\footnotetext{
${ }^{27}$ Vgl. Ingo Broer, Einleitung in das Neue Testament, Würzburg ${ }^{3} 2010,633$.

${ }^{28}$ Werbick, Gott-menschlich, 212.

${ }^{29}$ Dorothee Sölle, Das Fenster der Verwundbarkeit. Theologisch-politische Texte, Stuttgart 1987, 7 .
} 
die Wunden Jesu, aus denen Blut und Wasser strömte, ${ }^{30}$ sind es, die heilsame Kraft besitzen, sondern es ist die Liebe Gottes, die sich im verwundeten Jesu vergegenwärtigte und die sich im Kreuzesgeschehen als Totalhingabe an die Menschen zu deren Heil zeigt. Dies beweist einerseits die Solidarität Gottes mit den Verwundeten und zeigt, dass Gottes Liebe auch in den schlimmsten Situationen immer da ist. Das Blut und Wasser Jesu bedeutet im Kontext des sakralen Opfers (sacrifice), dass das heilige Blut und Wasser Jesu in Entsprechung zum jüdischen Sühneopferritual die Erde tränkt, reinigt und mit der Lebenskraft Gottes erneuert. Durch das Blut und Wasser Jesu ist die ganze Erde und die Menschheit mit göttlicher Kraft belebt und erneuert worden. Die Wunden Jesu haben Welt und Mensch aus den Konsequenzen der adamischen Schuld gerettet und damit Gott und Mensch versöhnt. Der neue Äon als Ermöglichung einer neuen Lebensgemeinschaft zwischen Gott und Mensch ist damit auf weltund religionsgeschichtlicher Ebene besiegelt. Das Blut der Wunden Jesu hat also Heilsrelevanz auf der Ebene der Heilsgeschichte als einer Geschichte zwischen Gott und der durch den Sündenfall gefallenen Menschscheit und ihrer Erde. Mit dem Blut des sacrifice ist demnach auch die Erde gemeint, die seitdem in den Geburtswehen der Neuschöpfung liegt (Röm 8, 22f.), da sie vom Ballast des alten Äons erlöst worden ist. Als Erstlingsgabe für diese sich im Prozess befindende Neuschöpfung erhalten die Gläubigen den Hl. Geist (Röm 8,23), der hilft, in der von Jesus eröffneten Gotteskindschaft zu leben und an dieser neuen Seinsqualität teilzuhaben.

Heilen und retten im starken Sinne tut aber Gottes Liebe, nicht die Wunden Jesu. Wunden können keine heilsamen Kräfte für andere Menschen in konkreten Notsituationen entwickeln. Legt man den Wunden Jesu theologisch eine heilsame Wirkung bei, wird die Verherrlichung des Opfers Jesu manifestiert, sodass als negative Folge davon die Opfer abermals auf ihr Verletztsein fixiert werden. Ihre Wunden könnten dabei schnell wieder religiös überhöht werden. Gerade im Interesse der Menschen, die auf der Verliererseite stehen, ist es sehr wichtig, an dieser Stelle genau zu differenzieren und zu analysieren, inwiefern und in Bezug worauf die Verwundungen Jesu, genauer gesagt sein Blut, Heilsrelevanz haben. Heilsrelevant für diese Menschen ist es, zu sagen, dass es eine Kraft hinter den Wunden Jesu gibt, die bereit war, in diesen physischen und psychischen Schmerz zu gehen, und die zugleich jene Kraft ist, die es vermag, Wunden

\footnotetext{
${ }^{30}$ Vgl. Werbick, Gott-menschlich, 212. Er deutet das Blut und Wasser, das in der Passionsgeschichte aus den Wunden Jesu fliesst (Joh 19, 30.34), als Metapher für Leben, Gnade und Versöhnung, das den Menschen zuströmt.
} 
zu heilen und vom Schmerz zu erlösen. Es ist die Kraft der Liebe Gottes. Sie ist auch das Motiv dafür, den Menschen aus seinem (selbst- und fremdverschuldeten) Leid zur erlösen. Diese Liebe ist mit Schopenhauers Diktum "Alle Liebe (agape, caritas) ist Mitleid « ${ }^{31}$ zu charakterisieren. Diese für Schopenhauer reine Liebe im Aspekt der agape bzw. caritas verknüpft er mit Mitleid. Mitleid definiert er als die »Erkenntnis fremden Leidens ${ }^{32}$. Die Erkenntnis fremden Leidens motiviert zum Handeln, um das Leid des Anderen zu lindern. Von daher ist mit Thomas Pröpper zu sagen: „Erlöst also hat uns die Liebe, die Jesus unter uns lebte und die sich, nachdem sie den Tod nicht scheute, als Gottes Liebe erwies. Und erlöst sind wir bereits in dem Glauben, dass Gott die Liebe selbst ist, dass sie verlässlich und noch den Sündern treu ist und ihre Möglichkeiten mit dem Tod nicht erschöpft sind. ${ }^{33}$ Gottes Liebe erweist sich im Kreuzes- und Auferweckungsereignis mächtiger als derTod und kann neues Leben schaffen. Für die Menschen heisst das, dass sie darauf setzen können, dass Gott auch in äussersten Lebenssituationen empathisch nahe ist und retten kann. Das menschliche Gefühl der Gottverlassenheit ist eine Illusion.

Die empathische Liebe Gottes kann sich im Hl. Geist vergegenwärtigen. Die rabbinischen Schekhina-Gleichnisse bezeugen auf eindrückliche Weise diese empathische Eigenschaft. Die Schekhina steht analog zur Ruach-Vorstellung im Alten Testament und lässt sich erst ab der Zeit der Tempelzerstörung durch die Römer (70 n. Chr.) als Ausdruck des Ringens um die Anwesenheit Gottes in einer katastrophalen Situation belegen. Talmud und Midrasch verstehen daher die Schekhina als die Immanenz Gottes in der Welt. ${ }^{34}$ Dieser Aspekt ist identisch mit dem Hl. Geist als Selbstvergegenwärtigung Gottes in der Welt und so kann die jüdische Schekhina-Vorstellung die Empathie des jüdisch-christlichen Gottes verdeutlichen: "Gott als Schekhina teilt die Ohnmacht, dieVerlassenheit und den Schmerz, die sein Volk durchlebt, und er lebt und leidet sie selbst durch. [...] Gott läßt seine Schekhina bei den Israeliten wohnen um ihrer Liebe willen. ${ }^{35}$ Die Einwohnung, das Mitgefühl und die Solidarität des Hl. Geistes vergegenwärtigen Gottes Liebe und wirken heilsam. Warum? " $\mathrm{Zu}$ wissen, dass wir nicht verlassen sind, ändert die Sache

\footnotetext{
${ }^{31}$ Arthur Schopenhauer, Die Welt als Wille und Vorstellung, $\$ 67$, in: Werke in zehn Bänden, Bd. 2, Züricher Ausgabe, Zürich 1977, 464.

32 Ebd.

${ }^{33}$ Pröpper, Erlösungsglaube und Freiheitsgeschichte, 97.

${ }^{34}$ Vgl. Hanspeter Ernst, Die Schekhina in rabbinischen Gleichnissen, Bern/Berlin u.a., 1993, 14 f.

${ }^{35}$ Ebd., 374.
} 
völlig. $.1^{36}$ Barmherzige Empathie "verlangt keinen Stoizismus, der Schmerz leugnet. Sie [die Barmherzigkeit, Vf.] beginnt dort, wo die Leidende ist - in der Trauer, Schande und Hoffnungslosigkeit. Sie nimmt die Verzweiflung als die wirklichste Realität wahr. [...] Diese Gemeinschaft mit der Leidenden in ihrem Schmerz, wie sie ihn erlebt, ist die Gegenwart der Liebe, die ein Balsam für die verwundete Seele ist. Diese Beziehung geteilten, mitfühlenden Leidens vermittelt Trost und Respekt, die Leidende ermächtigen können, den Schmerz zu ertragen, sich der Demütigung zu widersetzen, die Schande zu überwinden. $\aleph^{37}$ Ein neues Leben kann möglich werden. Jesus als stigmatisierter Auferstandener ist ein wirksames Beispiel für die Hoffnung, dass Gott im Leiden nahe ist und hilft, es zu überwinden. Es lohnt sich, auf dieses Beispiel zu setzen. Der Künstler Joseph Beuys hat dies getan. Er selbst, ein Kriegsopfer mit schwerer Verwundung am Kopf, ging durch eine Phase der Depression und erlebte eine Transformation, die er in seiner Kunst verarbeitete. Mit der Rauminstallation "zeige deine wunde« wollte er zum Ausdruck bringen, dass Heilung und Transformation nur geschehen kann, wenn man anfängt, der Wunde Aufmerksamkeit zu schenken und sie ohne Scham zu zeigen. ${ }^{38}$ Die eigentliche Herausforderung für einen Künstler sah er darin, das "geistige Geschehen, das todüberwindende Geheimnis des Christus darzustellen « ${ }^{39}$ Er sah keinen Sinn darin, nur bei Karfreitagsmotiven stehen zu bleiben. »Der Sinn des Christusthemas war der des Sieges, der Transformation, der Überwindung, der Hoffnung. $\star^{40}$

Im Angesicht des Auferstandenen mit den Wundmalen kann heute unter den Bedingungen der Kontingenz und des bereits angebrochenen neuen Äons begründet auf Heilung und neues Leben gehofft werden, kann die Transformation in einen vielleicht stigmatisierten

\footnotetext{
${ }^{36}$ Elizabeth A. Johnson, Ich bin die ich bin. Wenn Frauen Gott sagen, Düsseldorf 1994, 358.

${ }^{37}$ Wendy Farley, Tragic Vision and Divine Compassion. A Contemporary Theodicy, Louisville-Kentucky 1990, 81, zit. n. Johnson, Ich bin die ich bin, 358.

${ }^{38}$ In der Süddeutschen Zeitung vom 26./27. Januar 1980 erschien ein Artikel zu einem Gespräch mit Beuys über diese Rauminstallation. Dort erklärt Beuys: "Zeige deine Wunde, weil man die Krankheit offenbaren muss, die man heilen will. Der Raum [...] spricht von der Krankheit der Gesellschaft [...] Dann ist natürlich der traumatische Charakter angesprochen. Eine Wunde, die man zeigt, kann geheilt werden. Das Kunstwerk bleibe nicht bei der Verwundung stehen. Es enthalte, so Beuys, darüber hinaus `Andeutungen`, dass die Todesstarre überwunden werden kann [...] etwas $[\ldots]$, das, wenn man genau hinhört, einen Ausweg weist.«

${ }^{39}$ Friedhelm Mennekes, Joseph Beuys: Christus Denken - Thinking Christ, Stuttgart 1996, 95.

${ }^{40}$ Ebd.
} 
"Auferstehungsleib « und Versöhnung mit Gott und Mitmenschen geschehen. Die Kraft, die heilsam ist und eine Transformation bewirkt, ist aber der Hl. Geist, die schöpferische Liebes- und Lebenskraft Gottes. So hat Paulus letztlich recht, wenn er sagt, dass wir durch das Leben Christi gerettet wurden, nachdem wir durch den Tod am Kreuz, dem sacrifice, versöhnt worden sind (Röm 5,10). Und in der von Paulus geforderten Kreuzesnachfolge zu sein, bedeutet, unter den Bedingungen der Kontingenz im Geist Christi zu leben. Das ist jene neue Seinsqualität, die uns auch zu "Händen Gottes" in der Welt macht. Eine Seinsqualität, die nicht auf sich fixiert bleibt, sondern es vermag, Versöhnungsprozesse zu initiieren, verletzten Mitmenschen durch Empathie oder praktische Hilfe ein Stück von Gottes Barmherzigkeit zu vergegenwärtigen und das Gefühl von Verlassensein zu lindern. Kreuzesnachfolge bedeutet im Blick auf verwundete Flüchtlinge, sie in ihrer Not nicht allein zu lassen, das Herz zu öffnen und Gottes Liebe konkret werden lassen - Möglichkeiten dazu gibt es viele.

— Dr. theol. Martina Bär war bis Ende 2016 Oberassistentin am Institut für Hermeneutik und Religionsphilosophie. Jetzt arbeitet sie als Fachperson für interreligiösen Dialog in Bern (Haus der Religionen). Zu ihren Arbeitsgebieten gehören die Theologische Anthropologie und Gottesrede in spätmodernen Städten. 